\title{
Introduction to Hyperspaces
}

\author{
Mark Burgin \\ Department of Mathematics \\ UCLA \\ Los Angeles, USA
}

\begin{abstract}
The development of mathematics brought mathematicians to infinite structures. This process started with transcendent real numbers and infinite sequences going through infinite series to transfinite numbers to nonstandard numbers to hypernumbers. From mathematics, infinity came to physics where physicists have been trying to get rid of infinity inventing a variety of techniques for doing this. In contrast to this, mathematicians as well as some physicists suggested ways to work with infinity introducing new mathematical structures such distributions and extrafunctions. The goal of this paper is to extend mathematical tools for treating infinity by considering hyperspaces and developing their theory.
\end{abstract}

Keywords-vector space, hypervector, hyperspace, series, sequence, summation

\section{INTRODUCTION}

The basic structures of the standard calculus and functional analysis are constructed using infinite operations with numbers and functions such as convergence of sequences and summation of series $[1,2]$. The calculus and functional analysis have many applications beyond mathematics. For instance, summation of series is widely applied in physics (cf., for example, [3 - 5]. However, the classical summation methods provide sums not for all series of real or complex numbers. That is why mathematicians developed various techniques for extending the domain of summable series [6 8]. Examples are Cesàro, Abel or Borel summation, each of which extends the classical technique of series summability.

Similar problems of divergence emerged in applications of integration in theoretical physics [9, 3]. Although renormalization techniques allowed elimination of some divergencies of integrals and series, these methods did not give adequate solutions of these problems (cf., for example, [10]). Nonstandard analysis also was not very helpful in this respect [11].

Nevertheless, neither of the known generalized techniques allows summability of arbitrary series of real (complex) numbers. Only the extension of real (complex) numbers to real (complex) hypernumbers establishes universal summability of real (complex) numbers [12]. A similar construction of extrafunctions extended the scope and applications of distributions [13]. These constructions found utilization in different areas including probability theory and its applications [14] as well as integration theory in general and the theory of the Feynman integral in particular [15].
At the same time, the extension of numbers to hypernumbers does not provide summability of arbitrary series and convergence of arbitrary sequences of real (complex) hypernumbers. To achieve this, we need to make one more extension to the numerical hyperspaces over the space of real (complex) hypernumbers [16, 17].

Nevertheless, even this extension does provide sufficient tools for infinite operations such series summation for sequences of functions. That is why in this paper, we develop a theory of general hyperspaces. The structure and properties of general hyperspaces used for rigorous determination of irregular operations with hypernumbers and extrafunctions, as well as their application to the problems of summation of hypernumbers is studied and integration of extrafunctions based on hypermeasures [18] is considered.

\section{CONSTRUCTING HYPERSPACES}

Let us consider a real normed vector space $\boldsymbol{V}$ with a norm $\|\cdot\|$ and take the set $\boldsymbol{V}^{\omega}=\left\{\left(u_{n}\right)_{n \in \omega} ; u_{n} \in \boldsymbol{V}\right\}$ of all $\boldsymbol{V}$ sequences, i.e., sequences of elements from $\boldsymbol{V}$, where a sequence $\boldsymbol{u}=\left(u_{n}\right)_{n \in \omega}$ of elements from $\boldsymbol{V}$ is represented by a mapping $f_{a}: \omega \rightarrow \boldsymbol{V}$ and $\omega$ is the ordered set $\{1,2,3, \ldots, m, \ldots$ \} of all natural numbers. In what follows, we use two notations for sequences $\boldsymbol{u}=\left(u_{n}\right)_{n \in \omega}$ and $\boldsymbol{u}=\left\{u_{n} ; n=1,2,3, \ldots, m, \ldots\right\}$.

Definition 1. For arbitrary sequences $\boldsymbol{u}=\left(u_{n}\right)_{n \in \omega}$ and $\boldsymbol{v}=$ $\left(v_{n}\right)_{n \in \omega}$ from $\boldsymbol{V}^{\omega}$, the relation

$$
\boldsymbol{u} \approx \boldsymbol{v} \text { means that } \lim _{n \rightarrow \infty}\left\|u_{n}-v_{n}\right\|=0
$$

Lemma 1. The relation $\approx$ is an equivalence relation in $\boldsymbol{V}^{\omega}$.

Lemma 2. If in the sequences $\left(u_{n}\right)_{n \in \omega}$ and $\left(v_{n}\right)_{n \in \omega}$, almost all elements are the same, then these sequences are equivalent, i.e., $\left(u_{n}\right)_{n \in \omega} \approx\left(v_{n}\right)_{n \in \omega}$.

Definition 2. Classes of equivalent with respect to $\approx$ sequences from $\boldsymbol{V}^{\omega}$ are called hypervectors and the set of all hypervectors (equivalence classes) is denoted by $\boldsymbol{V}_{\omega}$ is called a hyperspace over the vector space $\boldsymbol{V}$.

From this perspective, each hypervector has the form

$$
\operatorname{Hv}_{\boldsymbol{F}}\left(u_{n}\right)_{n \in \omega}=\left\{\left(v_{n}\right)_{n \in \omega} \in \boldsymbol{V}^{\omega} ;\left(b_{n}\right)_{n \in \omega} \approx\left(u_{n}\right)_{n \in \omega}\right\}
$$

It is also possible to treat hypervectors not as classes but as names of these classes. In this case, $\operatorname{Hv}_{F}\left(u_{n}\right)_{n \in \omega}$ is a name of the class $\left\{\left(v_{n}\right)_{n \in \omega} \in \boldsymbol{V}^{\omega} ;\left(v_{n}\right)_{n \in \omega} \approx\left(u_{n}\right)_{n \in \omega}\right\}$ of sequences. 
Definition 2 defines the natural projection $p: \boldsymbol{V}^{\omega} \rightarrow \boldsymbol{V}_{\omega}$ where $p\left(\left(u_{n}\right)_{n \in \omega}\right)=\operatorname{Hv} F\left(u_{n}\right)_{n \in \omega}$.

Example 1. When $\boldsymbol{V}$ is the one-dimensional space $\boldsymbol{R}$ of all real numbers, then $\boldsymbol{V}_{\omega}$ is the space $\boldsymbol{R}_{\omega}$ of all real hypernumbers [12].

Example 2. When $\boldsymbol{V}$ is the space $\boldsymbol{C}$ of all complex numbers as vectors over $\boldsymbol{R}$, then $\boldsymbol{V}_{\omega}$ is the space $\boldsymbol{C}_{\omega}$ of all complex hypernumbers [17].

Example 3. When $\boldsymbol{V}$ is the space $\boldsymbol{F}$ of real functions, then $\boldsymbol{V}_{\omega}$ is the space $\boldsymbol{F}_{\omega}$ of all real extrafunctions [12]. It is necessary to note that the theory of extrafunctions is a far-reaching development of the theory of distributions [13]

According to Definition 2, any sequence $\boldsymbol{u}=\left(u_{n}\right)_{n \in \omega}$ with elements from $\boldsymbol{V}$ determines (and represents) a hypervector $\chi=$ $\operatorname{Hv}_{\boldsymbol{F}}\left(u_{n}\right)_{n \in \omega}$ where $\boldsymbol{u} \in \chi$.

Definition 3. The sequence $\boldsymbol{u}$ is called a defining sequence or representing sequence or representation of the hypervector $\chi$ $=\operatorname{Hv}_{F}\left(u_{n}\right)_{n \in \omega}$.

Definition 2 implies that any two hypernumbers $\operatorname{Hv} F\left(u_{n}\right)_{n \in \omega}$ and $\operatorname{Hv}_{F}\left(v_{n}\right)_{n \in \omega}$ are equal if and only if their defining sequences $\left(u_{n}\right)_{n \in \omega}$ and $\left(v_{n}\right)_{n \in \omega}$ are equivalent.

Note that by Lemma 2, any hypervector $\chi$ in $\boldsymbol{V}_{\omega}$ does not depend on a finite number of elements from its representation, i.e., we have the following result.

Lemma 3. If $\chi=\operatorname{Hv}_{F}\left(u_{n}\right)_{n \in \omega}$ and almost all elements in sequences $\left(u_{n}\right)_{n \in \omega}$ and $\left(v_{n}\right)_{n \in \omega}$ are the same (equal), then $\chi=$ $\operatorname{Hv} F\left(v_{n}\right)_{n \in \omega}$.

Definition 4. A sequence $\boldsymbol{u}=\left(u_{n}\right)_{n \in \omega}$ is called bounded if there is a positive real number $c$ such that $\left\|u_{n}\right\|<c$ for all $n \in \omega$.

Bounded vector sequences define bounded hypervectors.

Definition 5. A hypervector $\chi$ is called bounded if it has a bounded defining sequence.

Lemma 4. If a hypervector $\chi$ is bounded, then each of its defining sequences is bounded.

\section{PROPERTIES OF HYPERSPACES}

Let us obtain some properties of hyperspaces.

Taking a subset $P$ of a vector space $\boldsymbol{V}$, it is possible to define its $\omega$-extension $P_{\omega}$ as follows

$P_{\omega}=\left\{\chi \in V_{\omega} ;\right.$ there is a sequence $\left(u_{n}\right)_{n \in \omega}$ with all elements from $P$ and $\left.\chi=\operatorname{Hv}_{F}\left(u_{n}\right)_{n \in \omega}\right\}$

Let us consider some properties of $\omega$-extensions.

Proposition 1. If $P \subseteq V$, then $p\left(P^{\omega}\right)=P_{\omega}$.

$\omega$-extensions preserve inclusion of sets, i.e., we have the following result.

Proposition 2. If $Q \subseteq P \subseteq V$, then $Q_{\omega} \subseteq P_{\omega} \subseteq V_{\omega}$.

Let us consider set-theoretical operations with $\omega$ extensions.
Proposition 3. If $Q, P \subseteq V$, then $Q_{\omega} \cup P_{\omega} \subseteq(Q \cup P)_{\omega}$.

The following example shows that in a general case, the inclusion in Proposition 3 is proper.

Example 4. Let us take $Q=\{0\}$ and $P=\{1\} \subseteq \boldsymbol{R}$, which is considered as a one-dimensional vector space, then $Q_{\omega}$ contains only one element and $P_{\omega}$ contains only one element while ( $Q \cup$ $P)_{\omega}$ contains infinitely many elements. For instance, the sequence $\boldsymbol{u}=(0,1,0,1,0,1,0,1, \ldots)$ defines the hypervector that does not belong either to $Q_{\omega}$ or to $P_{\omega}$. Consequently, we have $Q_{\omega} \cup P_{\omega} \neq(Q \cup P)_{\omega}$.

Proposition 4. If $Q, P \subseteq V$, then $(Q \cap P)_{\omega} \subseteq Q_{\omega} \cap P_{\omega}$.

The following example shows that in a general case, the inclusion in Proposition 4 is proper.

Example 5. Let us take $Q=\left\{1 / 2^{n} ; n=2,3,4,5, \ldots\right\}$ and $P$ $=\left\{1 / 3^{n} ; n=2,3,4,5, \ldots\right\} \subseteq C$, then $Q \cap P$ is empty and consequently, $(Q \cap P)_{\omega}$ is empty. At the same time, $Q_{\omega}$ contains the hypervector $\operatorname{Hv}\left\{1 / 2^{n} ; n=2,3,4,5, \ldots\right\}=0$ and $P_{\omega}$ contains the hypervector $\operatorname{Hv}\left\{1 / 3^{n} ; n=2,3,4,5, \ldots\right\}=0$. We see that $Q_{\omega} \cap P_{\omega}$ is not empty containing the hypervector 0 $=\operatorname{Hv}(0,0, \ldots, 0)$ and consequently, $(Q \cap P)_{\omega} \neq Q_{\omega} \cap P_{\omega}$.

In some cases, sets $P_{\omega}$ and $V_{\omega}$ coincide demonstrating that $\omega$-extensions do not always preserve the strict inclusion relations of sets.

Theorem 1. If $P$ is a dense subset of $V$, then there is a oneto-one correspondence between the set $P_{\omega}$ and the set $\boldsymbol{V}_{\omega}$ of all hypervectors, i.e., these sets are equipotent.

Example 6. The set $Q$ of all rational numbers is a proper subset of the one-dimensional vector space $\boldsymbol{R}$ of all real numbers. However, by Theorem $1, Q_{\omega}=\boldsymbol{R}_{\omega}$.

Taking different norms in the space $\boldsymbol{V}$, it is possible to build different hyperspaces $\boldsymbol{V}_{\omega}$, which coincide only in some cases. It is possible to ask a question whether there is the largest hyperspace over $\boldsymbol{V}$. It exists and to build it, we need further development of the theory.

Definition 6. Two sequences $\boldsymbol{u}=\left(u_{n}\right)_{n \in \omega}$ and $\boldsymbol{v}=\left(v_{n}\right)_{n \in \omega}$ from $\boldsymbol{V}^{\omega}$ are cofinitely equivalent if almost all elements in $\boldsymbol{u}$ and $v$ are the same (equal).

It is possible to consider the factoring $\boldsymbol{V}_{\text {cofin }}$ of the set $\boldsymbol{V}^{\omega}$ by this equivalence. At the same time, any norm in a vector space defines a metric $[2,19]$. So, we can take the space $V$ with some metric $\mathbf{d}$ and build the hyperspace $\boldsymbol{V}_{\boldsymbol{\omega d}}$. This hyperspace has the following property.

Proposition 5. If the metric $\mathbf{d}$ in the vector space $\boldsymbol{V}$ defines the discrete topology in $\boldsymbol{V}$, then $\boldsymbol{V}_{\boldsymbol{\omega d}}=\boldsymbol{V}_{\text {cofin }}$.

It means that $\boldsymbol{V}_{\text {cofin }}$ is a hyperspace over $\boldsymbol{V}$.

Lemma 3 implies the following result.

Proposition 6. For any metric $\mathbf{d}$ in the vector space $\boldsymbol{V}$, there is a natural projection $q: \boldsymbol{V}_{\text {cofin }} \rightarrow \boldsymbol{V}_{\omega \mathbf{d}}$.

Corollary 1. For any normed vector space $\boldsymbol{V}$, there is a natural projection $q: \boldsymbol{V}_{\text {cofin }} \rightarrow \boldsymbol{V}_{\omega}$. 
This shows that $\boldsymbol{V}_{\text {cofin }}$ is the largest hyperspace over $\boldsymbol{V}$.

\section{OPERATIONS IN HYPERSPACES}

Operations in $F$ induce corresponding operations in $F^{\omega}$.

Let $\boldsymbol{u}=\left(u_{i}\right)_{i \in \omega}$ and $\boldsymbol{v}=(v)_{i \in \omega}$ be elements from $\boldsymbol{V}^{\omega}$ and assume $a$ is a real number. Then we have the following operations in $\boldsymbol{V}^{\omega}$.

Definition 7. a) Operation of addition in $\boldsymbol{V}^{\omega}$ is defined as $\boldsymbol{u}$ $+\boldsymbol{v}=\left(z_{i}\right)_{i \in \omega}$ where $z_{i}=u_{i}+v_{i}$ for all $i \in \omega$;

b) Operation of subtraction in $\boldsymbol{V}^{\omega}$ is defined as $\boldsymbol{u}-\boldsymbol{v}=$ $\left(w_{i}\right)_{i \in \omega}$ where $w_{i}=u_{i}-v_{i}$ for all $i \in \omega$;

c) Operation of multiplication by real numbers is defined in $\boldsymbol{V}^{\omega}$ by the formula $\boldsymbol{a} \cdot \boldsymbol{v}=\left(z_{i}\right)_{i \in \omega}$ where $z_{i}=a v_{i}$ where for all $i \in \omega$;

By the definition of addition, multiplication by real numbers and subtraction in the set $\boldsymbol{V}^{\omega}$, all laws of operations with real numbers, such as commutativity of addition, associativity of addition, and associativity of multiplication, are valid for corresponding operations with sequences from $\boldsymbol{V}^{\omega}$.

In particular, we have the following result.

Proposition 7. $V^{\omega}$ is a real vector space.

Let us consider bounded sequences in this space.

Proposition 8. a) The sum and difference of two bounded vector sequences are bounded.

b) Multiplication of a bounded vector sequence by a real number gives a bounded vector sequence.

Proposition 8 implies the following result.

Corollary 2. Bounded vector sequences form a subspace $\boldsymbol{B}^{\omega}$ of the vector space $\boldsymbol{V}^{\omega}$.

Relations between the spaces $\boldsymbol{V}^{\omega}$ and $\boldsymbol{V}_{\omega}$ imply the following result.

Proposition 9. Operations of addition and subtraction in $\boldsymbol{V}^{\omega}$ induce similar operations in $\boldsymbol{V}_{\omega}$ with all identities preserved.

Namely, if $\chi=\operatorname{Hv}_{F}\left(u_{n}\right)_{n \in \omega}$ and $\varsigma=\operatorname{Hv}\left(v_{n}\right)_{n \in \omega}$ are hypervectors, then we have $\chi+\varsigma=\operatorname{Hv}\left(u_{n}+v_{n}\right)_{n \in \omega}$ and $\chi-\varsigma=$ $\operatorname{Hv}\left(u_{n}-v_{n}\right)_{n \in \omega}$.

Proposition 10. Multiplication by real numbers in $\boldsymbol{V}^{\omega}$ induces a similar operation in $\boldsymbol{V}_{\omega}$ with all identities preserved.

Namely, if $\chi=\operatorname{HVF}\left(u_{n}\right)_{n \in \omega}$ is a hypervector and $a$ is a real number, then $a \chi=\operatorname{Hv}\left(a u_{n}\right)_{n \in \omega}$.

Propositions 9 and 10 imply the following result.

Theorem 2. $V_{\omega}$ is a real vector space.

A subset $P$ of a vector space $V$ is called balanced [2] if for any $0 \leq t \leq 1$, we have

$$
t P \subseteq P
$$

Theorem 3. If $P$ is a balanced subset of a vector space $\boldsymbol{V}$, then the set $P_{\omega}$ is a balanced subset of the vector space $V_{\omega}$.

Corollary 3. If $Q$ and $P$ are balanced subsets of a vector space $\boldsymbol{V}$, then $(Q \cap P)_{\omega}, Q_{\omega} \cap P_{\omega}, Q_{\omega} \cup P_{\omega}$ and $(Q \cup P)_{\omega}$ are balanced subsets of the vector space $V_{\omega}$.

A subset $P$ of a vector space $\boldsymbol{V}$ is called convex [2] if for any $0 \leq t \leq 1$, we have

$$
t P+(1-t) P \subseteq P
$$

Theorem 4. If $P$ is a convex subset of a vector space $\boldsymbol{V}$, then the set $P_{\omega}$ is a convex subset of the vector space $V_{\omega}$.

Corollary 4. If $Q$ and $P$ are convex subsets of a vector space $\boldsymbol{V}$, then $(Q \cap P)_{\omega}$ and $Q_{\omega} \cap P_{\omega}$, are convex subsets of the vector space $\boldsymbol{V}_{\omega}$.

Now let us look how arithmetical operations transform bounded hypervectors.

Proposition 11. a) The sum and difference of two bounded hypervectors are bounded.

b) Multiplication of a bounded hypervector by a real number gives a bounded hypervector.

Proposition 11 implies the following result.

Corollary 5. Bounded hypervectors form a subspace $\boldsymbol{B}_{\omega}$ of the vector space $\boldsymbol{V}_{\omega}$.

Let us consider arithmetical operations with $\omega$-extensions in real vector spaces. There are two such operations - addition and multiplication by real numbers [2].

Proposition 12. If $Q, P \subseteq \boldsymbol{V}$ and both $Q$ and $P$ contain the zero vector 0 , then $Q_{\omega}+P_{\omega} \subseteq(Q+P)_{\omega}$.

The following example shows that in a general case, this inclusion is proper.

Example 7. Let us take subsets $Q=\{0,2\}$ and $P=\{0,1\}$ of the one-dimensional vector space $\boldsymbol{R}$, then neither $Q_{\omega}$ nor $P_{\omega}$ contain the element $3=1+2$ from $(Q+P)_{\omega}$. Consequently, we have $Q_{\omega}+P_{\omega} \neq(Q+P)_{\omega}$.

Note that having the zero vectors in both subsets is an essential condition in Proposition 12 because otherwise the inclusion of set can be invalid.

Proposition 13. If $P \subseteq V$ and $a$ is a real number, then $a(P)_{\omega}$ $=(a P)_{\omega}$.

An interesting question is whether it is possible to extend the norm in $\boldsymbol{V}$ to the norm in $\boldsymbol{V}_{\omega}$. However, we can show that in a general case even if $\boldsymbol{V}$ is a Euclidean space, $\boldsymbol{V}_{\omega}$ is not a normed space. At the same time, it is possible to prove the following result utilizing the concept of a hypernorm [17].

We remind that a hypernorm in a vector space $\boldsymbol{X}$ is a function $v: \boldsymbol{X} \rightarrow \boldsymbol{R}_{\omega}{ }^{+}$, which satisfies the following conditions:

N1. For any $x$ from $L, q(x)=0$ if and only if $x=\mathbf{0}$.

N2. $q(a x)=\|a\| \cdot q(x)$ for any $x$ from $L$ and any element $a$ from $\boldsymbol{F}$. 
N3 (the triangle inequality or subadditivity).

$$
q(x+y) \leq q(x)+q(y) \text { for any } x \text { and } y \text { from } L
$$

A vector space $L$ with a hypernorm is called a hypernormed vector space or simply, a hypernormed space.

The real hypernumber $q(x)$ is called the hypernorm of the element $x$ from the hypernormed space $L$.

Note that norms in vector spaces coincide with those hypernorms that take values only in the set of real numbers. That is, we have the following result [17].

Proposition 14. A hypernorm is a norm in a vector space if and only if it takes values in the set of all non-negative real numbers.

Thus, any normed vector space is a hypernormed vector space. Besides, any hypernormed vector space is a hyperseminormed vector space.

Theorem 5. $V_{\omega}$ is a hypernormed real vector space.

Taking a hypervector $\chi=\operatorname{Hv} F\left(u_{n}\right)_{n \in \omega}$, we define its hypernorm as the hypernumber a $\alpha=\operatorname{Hv}_{F}\left(\left\|u_{n}\right\|\right)_{n \in \omega}$.

In normed vector spaces, the norm defines a metric [2, 19]. In a similar way, the hypernorm defines a hypermetric in hypernormed vector spaces [17].

We remind that a mapping $\mathbf{d}: X \times X \rightarrow \boldsymbol{R}_{\omega}{ }^{+}$is called a hypermetric (or a hyperdistance function) in a set $X$ if it satisfies the following axioms:

M1. For any $x$ and $y$ from $X, \mathbf{d}(x, y)=0$ if and only if $x=$ $y$.

M2 (Symmetry). $\mathbf{d}(x, y)=\mathbf{d}(y, x)$ for all $x, y \in X$.

M3 (the triangle inequality or subadditivity).

$x, y, z \in X$.

$$
\mathbf{d}(x, y) \leq \mathbf{d}(x, z)+\mathbf{d}(z, y) \text { for all }
$$

A set $X$ with a hypermetric $\mathbf{d}$ is called a hypermetric space.

The real hypernumber $\mathbf{d}(x, y)$ is called the distance between $x$ and $y$ in the hypermetric space $X$.

Thus, Theorem 5 implies the following result.

Theorem 6. $V_{\omega}$ is a hypermetric space.

A metric defines a topology in the metric space $[2,19]$. In a similar way, hypermetric also defines a topology in the hypermetric space [17]. Consequently, we have the following result.

Theorem 7. $V_{\omega}$ is a topological space.

Topology in $\boldsymbol{V}_{\omega}$ is defined by the system of neighborhoods of 0 having the form

$U_{a}=\left\{\chi=\operatorname{Hv}_{\boldsymbol{F}}\left(u_{n}\right)_{n \in \omega} ;\|\chi\|<a\right\}$

where $a$ is a positive real number.
A subset $P$ of a vector space $\boldsymbol{V}$ is called bounded [2] if for any neighborhood $U_{a}$ of 0 , there is a positive real number $t$ such that $P \subseteq t U_{a}$.

Theorem 8. If $P$ is a bounded subset of a vector space $\boldsymbol{V}$, then the set $P_{\omega}$ is a bounded subset of the vector space $V_{\omega}$.

Corollary 6. If $Q$ and $P$ are bounded subsets of a vector space $\boldsymbol{V}$, then $(Q \cap P)_{\omega}, Q_{\omega} \cap P_{\omega}, Q_{\omega} \cup P_{\omega}$ and $(Q \cup P)_{\omega}$ are bounded subsets of the vector space $\boldsymbol{V}_{\omega}$.

Theorem 8 and Propositions 11 and 12 imply the following result

Corollary 7. If $Q$ and $P$ are bounded subsets of a vector space $\boldsymbol{V}$, then $Q_{\omega}+P_{\omega},(Q+P)_{\omega}$ and $(a P)_{\omega}$ are bounded subsets of the vector space $V_{\omega}$.

In a topological vector space, any compact set is bounded [19]. In contrast to this, in the hyperspace $\boldsymbol{V}_{\omega}$, even one hypervector can be unbounded. For instance, the hypervector (hypernumber) $\chi=\operatorname{Hv}_{F}(n)_{n \in \omega}$ is unbounded in the hyperspace $\boldsymbol{R}_{\omega}$. Thus, for hyperspaces, we need a more general definition of boundedness.

Let us consider an element $u$ in a real vector space $\boldsymbol{U}$.

Definition 8. A subset $P$ of the space $\boldsymbol{U}$ is called $u$-bounded if for every neighborhood $U$ of $u$, there is a real number $a$ such that $P \subseteq a U$.

This definition implies the following result.

Lemma 5. A subset $P$ of the space $\boldsymbol{U}$ is bounded if and only if it is 0-bounded.

For normed vector spaces, the concepts of boundedness and $u$-boundedness coincide.

Lemma 6. If $U$ is a normed vector space and $u \in U$, then any $u$-bounded in $\boldsymbol{U}$ subset $P$ is bounded.

In hyperspaces, this is not true in a general case.

Proposition 15. A $\chi$-bounded subset $P$ of the space $\boldsymbol{U}$ is bounded if and only if the hypervector $\chi$ is bounded.

The following result generalizes Corollary 6 .

Proposition 16. If $Q$ and $P$ are $\chi$-bounded subsets of a vector space $\boldsymbol{V}$, then $(Q \cap P)_{\omega}, Q_{\omega} \cap P_{\omega}, Q_{\omega} \cup P_{\omega}$ and $(Q \cup$ $P)_{\omega}$ are $\chi$-bounded subsets of the vector space $V_{\omega}$.

Note that the union of a $\chi$-bounded subset and $\varsigma$-bounded subset of the vector space $\boldsymbol{V}_{\omega}$ can be neither $\chi$-bounded nor $\varsigma$ bounded. However, Proposition 16 and Corollary 6 imply the following result.

Proposition 17. If the hypervectors $\chi$ and $\varsigma$ are bounded, then the union of a $\chi$-bounded subset and $\varsigma$-bounded subset of the vector space $\boldsymbol{V}_{\omega}$ is bounded.

A similar result is true for addition of sets in hyperspaces.

Proposition 18. If $Q$ and $P$ are $\chi$-bounded subsets of a vector space $\boldsymbol{V}$, then $Q_{\omega}+P_{\omega}$ and $(Q+P)_{\omega}$ are $2 \chi$-bounded subsets of the vector space $\boldsymbol{V}_{\omega}$. 
Note that the sum of a $\chi$-bounded subset and $\varsigma$-bounded subset of the vector space $\boldsymbol{V}_{\omega}$ can be neither $\chi$-bounded nor $\varsigma$ bounded. However, Proposition 16 and Corollary 6 imply the following result.

Proposition 19. If the hypervectors $\chi$ and $\varsigma$ are bounded, then the sum of a $\chi$-bounded subset and $\varsigma$-bounded subset of the vector space $\boldsymbol{V}_{\omega}$ is bounded.

An important class of vector spaces is formed by topological vector spaces. As Rudin writes, the concept of topological vector spaces pervades all of functional analysis [2]. However, in a general case, $\boldsymbol{V}_{\omega}$ is not a topological vector space although it is proved that any hypernormed real vector space is a semitopological vector space [17]. This gives us the following result.

Corollary 8. $\boldsymbol{V}_{\omega}$ is a semitopological vector space.

Note that although $\boldsymbol{V}_{\omega}$ is not a topological vector space in a general case, $\boldsymbol{V}_{\omega}$ is a topological space because a hypernorm defines a topology in a vector space [17].

Many properties of the real vector spaces $\boldsymbol{V}$ and $\boldsymbol{V}_{\omega}$ are closely related. In particular, the space $\boldsymbol{V}_{\omega}$ of hypervectors is a natural extension of the vector space $\boldsymbol{V}$ as we have the following result.

Theorem 9. The vector space $\boldsymbol{V}$ is isomorphic to the subspace $\underline{\boldsymbol{V}}=\left\{\chi=\operatorname{Hv}_{\boldsymbol{F}}\left(u_{n}\right)_{n \in \omega} ; u_{n}=u \in \boldsymbol{V}\right.$ for all $\left.n \in \omega\right\}$ of the vector space $V_{\omega}$

Identifying elements from $\boldsymbol{V}$ with stable sequences, i.e., sequences in which all elements are equal to the same element from $\boldsymbol{V}$, we can treat the vector space $\boldsymbol{V}$ as a subset of the set $\boldsymbol{V}_{\omega}$ of all hypervectors.

The norm in $\boldsymbol{V}$ is a restriction of the hypernorm in $\boldsymbol{V}_{\omega}$. This implies the following result.

Corollary 9. The normed vector space $\boldsymbol{V}$ is (or more exactly, is isomorphic to) a normed subspace of the hypernormed real vector space $\boldsymbol{V}_{\omega}$.

Let us consider $\omega$-extensions of subsets of the real normed vector space $\boldsymbol{V}$.

Proposition 20. a) If a subset $P$ of the vector space $V$ is closed with respect to addition, then $P_{\omega}$ is also closed with respect to addition.

b) If a subset $P$ of the vector space $V$ is closed with respect to subtraction, then $P_{\omega}$ is also closed with respect to subtraction.

c) If a subset $P$ of the vector space $V$ is closed with respect to multiplication by real numbers, then $P_{\omega}$ is also closed with respect to multiplication by real numbers.

Proposition 20 implies the following result.

Theorem 10. If $\boldsymbol{U}$ is a vector subspace of a normed vector space $\boldsymbol{V}$, then $\boldsymbol{U}_{\omega}$ is a vector subspace of $\boldsymbol{V}_{\omega}$.

The inclusion relation is preserved by $\omega$-extensions.
Proposition 21. For any subspace $\boldsymbol{W}$ of a subspace $\boldsymbol{U}$ of the space $\boldsymbol{V}$, the vector space $\boldsymbol{W}_{\omega}$ is a subspace of $\boldsymbol{U}_{\omega}$.

However, the strict inclusion relation is not always preserved by $\omega$-extensions as the following result demonstrates.

Let $\boldsymbol{U}$ be a dense subspace of $\boldsymbol{V}$.

Theorem 11. The vector spaces $\boldsymbol{U}_{\omega}$ and $\boldsymbol{V}_{\omega}$ are isomorphic.

There are natural relations between linear mappings of vector spaces and linear mapping of their hyperspaces.

Proposition 22. A linear mapping of a vector space $\boldsymbol{W}$ into a vector space $\boldsymbol{U}$ can be extended to a linear mapping of the vector space $\boldsymbol{W}_{\omega}$ into the vector space $\boldsymbol{U}_{\omega}$.

There are natural relations between linear functionals in vector spaces and linear hyperfunctionals in their hyperspaces.

We remind that a real hyperfunctional in a real vector space $\boldsymbol{L}$ is a mapping $F: \boldsymbol{L} \rightarrow \boldsymbol{R}_{\omega}[17]$.

Proposition 23. A linear functional in a vector space $\boldsymbol{V}$ can be extended to a linear hyperfunctional in the vector space $\boldsymbol{V}_{\omega}$.

Completeness is an important property of normed vector spaces $[1,2]$.

Theorem 12. For any real normed vector space $\boldsymbol{V}$, the vector space $\boldsymbol{V}_{\omega}$ contains a completion of the space $\boldsymbol{V}$.

Corollary 10. If $\boldsymbol{U}$ is a Banach space that contains $\boldsymbol{V}$, then there is a linear mapping of $\boldsymbol{U}$ into $\boldsymbol{V}_{\omega}$.

It would be interesting to know whether a proper counterpart of the Hahn-Banach theorem is true for hyperspaces.

Completeness properties of hyperspaces allow proving the following result.

Theorem 13. a) Any series $\boldsymbol{s}$ of vectors from the space $\boldsymbol{V}$ has the sum in the space $\boldsymbol{V}_{\omega}$.

b) If a series $\boldsymbol{s}$ of vectors from the space $\boldsymbol{V}$ has the classical sum $u$ in $\boldsymbol{V}$, then its sum in the space $\boldsymbol{V}_{\omega}$ coincides with $u$.

This shows that summation of vector series in hyperspaces is a natural extension of the conventional summation of vector series.

Summation in hyperspaces provides the base for integration in these spaces. However, to define integrals, it is necessary to consider hypermeasures in the sense of [18].

Let us consider some properties of summation related to operations with vectors and hypervectors. Sequences and series of vectors from the space $\boldsymbol{V}$ belong to the vector space $\boldsymbol{V}^{\omega}$. So, it is possible to perform operations of addition and multiplication by real numbers with them.

Theorem 14. a) If a hypervector $\chi$ is the sum of a series $s$ of vectors from the space $\boldsymbol{V}$ and $\varsigma$ is the sum of a series $\boldsymbol{r}$ of vectors from the space $\boldsymbol{V}$, then $\chi+\varsigma$ is the sum of a series $\boldsymbol{s}+\boldsymbol{r}$ in the space $\boldsymbol{V}_{\omega}$. 
b) If a hypervector $\chi$ is the sum of a series $s$ of vectors from the space $\boldsymbol{V}$ and $a$ is a real number, then $a \chi$ is the sum of a series $a \boldsymbol{s}$ in the space $\boldsymbol{V}_{\boldsymbol{\omega}}$.

This shows that summation commutes with operations in hyperspaces.

\section{CONCLUSION}

We see that hyperspaces make vector spaces complete and allowing to assign the sum to any vector series. This provides for effective application of these spaces in physics where series and their summation play a very important role. The next step is to study integration in hyperspaces. The results of integration with hypernumbers obtained in $[12,15,16]$ show that integration in hyperspaces can essentially extend the scope and applications of the integration in the initial vector space.

A broader problem is the development of functional analysis in hyperspaces. Specifically, it is important to find general counterparts of the basic principles of linear functional analysis for hyperspaces [19]. The goal is to bring new tools to the theory of operator equations in general and differential equations in particular making possible to solve much more equations than it is possible by traditional methods including distributions.

One more interesting problem for the future study is exploration of the categories of hyperspaces as well as additional algebraic structures related to hyperspaces. For instance, it is possible to consider linear hyperalgebras, i.e., hyperspaces with multiplication, and linear $\Omega$-hyperalgebras, i.e., hyperspaces with an additional system $\Omega$ of operations.

\section{REFERENCES}

[1] W. Rudin, Principles of Mathematical Analysis, McGraw-Hill, New York, 1976

[2] W. Rudin, Functional Analysis, McGrow-Hill, New York, 1991

[3] F. Close, The Infinity Puzzle: Quantum Field Theory and the Hunt for an Orderly Universe, Basic Books, New York, 2011

[4] J.C. Collins, Renormalization, Cambridge University Press, Cambridge, 1984

[5] K. Knopp, Theory and Application of Infinite Series , Dover, NY, 1940

[6] G.H. Hardy, Divergent Series, Oxford University Press, Oxford, 1949

[7] D.E. Loeb and G.-C. Rota," Formal Power Series of Logarithmic Type," Advances in Math., vol. 75, pp. 1-118, 1989

[8] G.G. Lorentz, "A contribution to the theory of divergent sequences," Acta Math., vol. 80, pp. 167-190, 1948

[9] R. P. Feynman and A. R. Hibbs, Quantum Mechanics and Path Integrals, McGraw-Hill Companies, 1965

[10] R. P. Feynman, QED, The Strange Theory of Light and Matter, Penguin Books, London, 1985

[11] A. Robinson, Nonstandard Analysis, Studies of Logic and Foundations of Mathematics, North-Holland, New York, 1966

[12] M. Burgin, Hypernumbers and Extrafunctions: Extending the Classical Calculus, Springer, New York, 2012

[13] L. Schwartz, Théorie des Distributions, Vol. I-II, Hermann, Paris, $1950 / 1951$

[14] M. Burgin and A.C. Krinik, "Hyperexpectations and random variables without expectations," Integration: Mathematical Theory and Applications, vol. 3, No. 3, pp. 245-267, 2012

[15] M. Burgin, "Hyperintegration approach to the Feynman integral," Integration: Mathematical Theory and Applications, vol. 1, No. 1, pp. 59-104, 2008

[16] M. Burgin, "Operations with Extrafunctions and Integration in Bundles with a Hyperspace Base," in Functional Analysis and Probability, Nova Science Publishers, New York, 2015, pp. 3 - 76

[17] M. Burgin, Semitopological Vector Spaces: Hypernorms, Hyperseminorms and Operators, Apple Academic Press, Toronto, Canada, 2017

[18] M. Burgin, "Hypermeasures in General Spaces," International Journal of Pure and Applied Mathematics, v. 24, No. 3, pp. 299-323, 2005

[19] N. Dunford and J. Schwartz, Linear Operators, Interscience Publishers, New York, 1958

\section{Creative Commons Attribution License 4.0 (Attribution 4.0 International, CC BY 4.0)}

This article is published under the terms of the Creative Commons Attribution License 4.0

https://creativecommons.org/licenses/by/4.0/deed.en US 